\title{
Improved Anti-Biofouling Performance of Thin -Film Composite Forward-Osmosis Membranes Containing Passive and Active Moieties
}

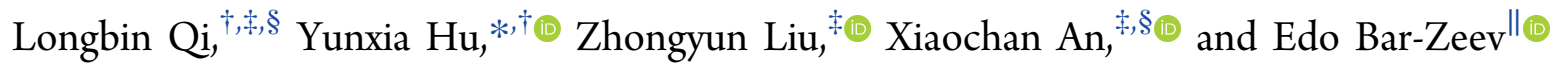 \\ ${ }^{\dagger}$ State Key Laboratory of Separation Membranes and Membrane Processes, School of Materials Science and Engineering, Tianjin \\ Polytechnic University, Tianjin 300387, PR China \\ ¥CAS Key Laboratory of Coastal Environmental Processes and Ecological Remediation; Research Center for Coastal Environmental \\ Engineering and Technology of Shandong Province; Yantai Institute of Coastal Zone Research, Chinese Academy of Sciences, \\ Yantai, Shandong Province 264003, PR China \\ ${ }^{\S}$ University of Chinese Academy of Sciences, Beijing 100049, PR China \\ "Department of Environmental Hydrology \& Microbiology, Zuckerberg Institute for Water Research (ZIWR), Ben-Gurion \\ University of the Negev, Beersheba, 8499000 Israel
}

\section{Supporting Information}

\begin{abstract}
Forward osmosis (FO) has gained increasing attention in desalination, wastewater treatment, and power generation. However, biofouling remains a major obstacle for the sustainable development of the FO process. Both passive and active strategies have been developed to mitigate membrane biofouling. A comprehensive understanding of different strategies and mechanisms has fundamental significance for the antifouling membrane development. In this study, thin-film composite (TFC) FO membranes were modified with polydopamine (PDA) coating as a passive antibacterial moiety and silver nanoparticles (Ag NPs) as an active antibacterial moiety. Their anti-biofouling performances were investigated both in static and dynamic conditions. In static exposure, the PDA-coated membranes exhibited great passive anti-adhesive property, and the Ag-NP-generated membranes presented both of excellent passive anti-adhesive properties and active antibacterial performance. While in dynamic cross-flow running conditions, Ag NPs effectively mitigated the membrane water flux decline due to their inhibition of biofilm growth, the PDA coating failed because of its inability to inactivate the attached bacteria growth. Moreover, Ag NPs were stable and active on membrane surfaces after $24 \mathrm{~h}$ of cross-flow operation. These findings provide new insights into the performances and mechanisms of passive and active moieties in the FO process.
\end{abstract}

\section{INTRODUCTION}

Increasing demand on freshwater and clean energy is one of the greatest global challenges due to the rapidly population growth, growing urbanization, and changing global climate. ${ }^{1,2}$ The forward-osmosis (FO) membrane process works as a state-of-the-art technology to desalinate seawater, ${ }^{3,4}$ reuse wastewater, $^{5-7}$ and produce clean energy using salinity gradients, ${ }^{8,9}$ alleviating freshwater shortages and clean energy crises locally and globally. The development of high-performance thin-film composite (TFC) FO membranes has gained increasing attention in the pursuit of achieving high water flux without compromising salt rejection during long-term operation under environmental conditions. ${ }^{10-12}$ However, membrane fouling (and particularly biofouling) remains a bottleneck for the development and application of large-scale FO systems, which significantly reduces membrane perform- ance and increases operation costs as well as energy inputs. $^{13-15}$

Passive and active strategies have been developed to mitigate biofilm by minimizing the deposition and growth of microorganisms on membrane surface. ${ }^{16-18}$ The passive strategy generally involves membrane surface modification by grafting, ${ }^{19-21}$ coating, or blending hydrophilic materials ${ }^{22,23}$ to weaken the foulant-membrane interactions and prevent foulants adsorption on membrane surface. ${ }^{16,24}$ The active strategy normally incorporates biocides on membrane surface to deactivate bacteria and prevent biofilm formation. ${ }^{25-27}$ Foulant adsorption on membrane surface is an initial stage of

Received: December 11, 2017

Revised: July 19, 2018

Accepted: August 3, 2018

Published: August 3, 2018 
membrane fouling. The passive strategy presents effective performances against fouling under low or mild fouling conditions. However, under severe fouling conditions, the passive strategy may lose its efficacy because the membrane surface is quickly masked by the foulant layer, and the foulantfoulant interaction is dominant to cause membrane fouling. ${ }^{28,29}$ Moreover, biofouling is more severe and complicated than inorganic and organic fouling because microorganisms are living foulants to secrete sticky proteins and polymers and induce strong interaction with membrane surfaces. ${ }^{28}$ The membrane performances may not be impaired if the attached microorganisms can be deactivated by biocides and then washed off easily. Therefore, an ideal anti-biofouling FO membrane should have both passive and active functions to minimize bacterial absorption and deactivate bacterial growth on the membrane surface. ${ }^{30} \mathrm{~A}$ holistic understanding of different strategies and mechanisms has fundamental significance for the antifouling membrane design.

However, few strategies have been developed to incorporate both passive and active moieties on membrane surfaces for the fabrication of ideal anti-biofouling FO membranes. Our previous work and other reported research have demonstrated that mussel-inspired dopamine chemistry provides a simple yet universal approach to incorporating both passive and active moieties on most types of inorganic and organic substrates. ${ }^{31-33}$ Dopamine can self-polymerize into polydopamine (PDA) on various substrates with controllable film thickness and durable stability. ${ }^{34}$ PDA has been confirmed to exhibit antifouling properties of alleviating the absorption of protein and microorganism. ${ }^{35,36}$ Silver nanoparticles (Ag NPs) can form in situ on the PDA coating upon incubation with silver salt solution and act as an efficient biocide. ${ }^{33,37-39}$ The $\mathrm{O}$ and $\mathrm{N}$ sites of PDA can serve as anchors for the Ag NPs to enhance their stability on membrane. Furthermore, Ag NPs are rechargeable on the PDA-coated surfaces and have exhibited sustainable bactericidal efficacy. ${ }^{33}$ Thus, dopamine chemistry provides a facile process by which to incorporate both passive PDA coating and active Ag NPs on FO membrane surfaces for anti-biofouling properties. However, no work has been done to investigate and compare the passive and active anti-biofouling performances of PDA and Ag-NP-functionalized TFC FO membranes in the continuous cross-flow forward-osmosis test system.

Herein, the thin-film composite (TFC) FO membranes were fabricated and then modified with PDA coating as a passive antibacterial moiety and Ag NPs as an active antibacterial moiety. Their anti-biofouling performances were investigated in both static and dynamic conditions and also compared on both operation modes of FO (active-layer-facing feed solution) and pressure-retarded osmosis (PRO, active layer facing draw solution) to gain comprehensive an understanding of the antibiofouling performances induced by PDA and Ag NPs. Pseudomonas aeruginosa was selected as a model bacterial stain, and synthetic wastewater was used as a low osmotic feed solution. Dynamic biofouling experiments were conducted in a continuous cross-flow FO system. The structure and composition of biofilms were analyzed to clarify the mechanisms of biofouling mitigation by PDA and Ag NPs. Our findings aim to provide new insights on the performances and mechanisms of passive and active moieties on the antibiofouling efficiency of TFC FO membranes.

\section{MATERIALS AND METHODS}

Materials and Chemicals. A commercial polyester nonwoven fabric (PET, grade 3249, $40 \mu \mathrm{m}$ thickness) was purchased from Ahlstrom (Helsinki, Finland). Polysulfone (PSf, Mn: $22000 \mathrm{Da}$ ), m-phenylenediamine (MPD, >99\%), 1,3,5-benzenetricarbonyl trichloride (TMC, 98\%), N-methyl pyrrolidone (NMP), and dopamine were supplied by SigmaAldrich (Saint Louis, MO). SYTO9 green fluorescent nucleic acid stain and Concanavalin A (Con A, Alexa Flour 633) were obtained from Invitrogen (Eugene, OR), and propidium iodide (PI) was received from Sigma-Aldrich. Tris-HCl buffer $(1 \mathrm{M}$, $\mathrm{pH}$ 8.5) was purchased from Beijing Solarbio Science \& Technology Co., Ltd.. P. aeruginosa (ATCC 27853) was obtained from the American Type Culture Collection. Yeast extract, tryptone, and agar were purchased from Oxoid Limited. $\mathrm{AgNO}_{3}, \mathrm{NaCl}, \mathrm{MgSO}_{4} \cdot 7 \mathrm{H}_{2} \mathrm{O}, \mathrm{NaHCO}_{3}, \mathrm{CaCl}_{2}$. $\mathrm{H}_{2} \mathrm{O}, \mathrm{KH}_{2} \mathrm{PO}_{4}, \mathrm{NH}_{4} \mathrm{Cl}, \mathrm{EDTA}$, and sodium citrate were purchased from Sinopharm Chemical Reagent Beijing Co., Ltd., China. All chemicals were used as received.

TFC Membrane Fabrication. TFC FO membranes were fabricated following our previous protocol through two steps including the casting of a PSf ultra-filtration support layer by a typical non-solvent-induced phase separation (NIPS) method and the formation of polyamide active layer by interfacial polymerization on top of PSf support. ${ }^{40,41}$ PSf casting solution (12 wt \%) in NMP was cast with a steel casting knife $(150 \mu \mathrm{m}$ gate height) on a clean glass plate covered by PET nonwoven fabric and precipitated in a water coagulation bath at room temperature to produce a PET-integrated PSf support membrane. During the casting process, the humidity was controlled at $40 \pm 5 \mathrm{RH} \%$ in a clean room. Polyamide layer was formed by interfacial polymerization of MPD in the aqueous phase and TMC in the organic phase on top of the PSf support layer. A wet PSf membrane was taped to a clean glass plate and then immersed in $30 \mathrm{~mL}$ of aqueous solution with 3.4 wt \% MPD for 2 min. A rubber roller was used to remove excess MPD solution from the PSf membrane surface. The MPD-containing PSf membrane was then immersed in a 0.15 wt \% TMC solution in hexane for $1 \mathrm{~min}$ to form polyamide films. Finally, the membranes were cured in DI water at $95{ }^{\circ} \mathrm{C}$ for $2 \mathrm{~min}$. After thorough rinsing with DI water, the TFC FO membranes were stored in DI at $4{ }^{\circ} \mathrm{C}$ before characterization.

TFC Membrane Surface Functionalization. In situ growth of Ag NPs on both surfaces of TFC FO membranes was performed according to our previous work. ${ }^{33}$ Briefly, the TFC FO membrane coupons were immersed in a $2 \mathrm{mg} / \mathrm{mL}$ dopamine solution at $10 \mathrm{mM}$ Tris- $\mathrm{HCl}$ buffer $(\mathrm{pH} 8.5)$ for 1 $\mathrm{h}$ at room temperature under $60 \mathrm{rpm}$ shaking to fabricate PDA-coated membranes. To generate Ag NPs on membrane surfaces, the PDA-coated membrane coupons were immersed in $50 \mathrm{mM}$ silver nitrate aqueous solution at room temperature, and the incubation time was varied $(1,2,4$, or $8 \mathrm{~h})$ to optimize the generation conditions of Ag NPs. Finally, the membrane coupons were rinsed three times with DI water, air-dried at room temperature, and stored in a light-proof dark chamber for further characterization.

TFC Membrane Characterization. The surface morphologies of pristine and modified membranes were observed using a scanning electron microscope (SEM, S-4800, Hitachi). Ag NPs on the membrane surfaces were detected using an EX-350 energy-dispersive X-ray microanalyzer (EDX, Horiba, Tokyo, 
Japan) with $15.0 \mathrm{kV}$ accelerating voltage. All samples were coated with $10 \mathrm{~nm}$ thick platinum $(\mathrm{Pt})$ for $100 \mathrm{~s}$ using an EMITECH SC7620 sputter coater before SEM and EDX observation. The particle size and number of Ag NPs were measured using Nano Measurer software and ImageJ software, respectively. The static water contact angles of membranes were measured using the sessile drop method on an optical instrument (OCA 20, Data Physics, Germany). Each sample (1 $\mathrm{cm} \times 5 \mathrm{~cm})$ was dried in a vacuum oven overnight and then mounted on a clean glass slide. A $2 \mu \mathrm{L}$ deionized (DI) water droplet was dropped on membrane surface using a microsyringe, and $2 \mathrm{~s}$ later, the droplet shape was recorded and analyzed using the automated Drop Shape Analysis software (SCA20 version 2 ). The surface $\zeta$ potential of membranes was measured using an Anton Paar SurPASS electrokinetic analyzer (Anton Paar, Austria) using $1.0 \mathrm{mM} \mathrm{KCl}$ solution as the background at $\mathrm{pH}$ of 7.5 . The $\zeta$ potential was determined using the associated software from the streaming potential slope versus pressure plots based on the HelmholtzSmoluchowski approach. All measurements were performed on at least three individual samples, and the data were reported as the average value and the standard deviation.

Static Anti-Adhesion Tests. P. aeruginosa was used as a model bacterial strain to evaluate the bacteria adhesion and viability on both surfaces of the TFC FO membrane, following our previous protocol with slight changes. ${ }^{42} P$. aeruginosa was initially cultured in Luria-Bertani (LB) broth to reach a midexponential growth phase. Bacteria were collected after $1 \mathrm{~min}$ of centrifugation at $5000 \mathrm{rpm}$ and then resuspended in sterile synthetic wastewater solution to reach an optical cell density at $600 \mathrm{~nm}\left(\mathrm{OD}_{600}\right)$ of 0.15 , where the initial concentration of $P$.

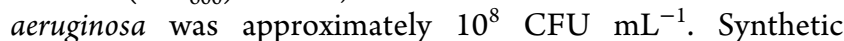
wastewater composed of $8.0 \mathrm{mM} \mathrm{NaCl}, 0.15 \mathrm{mM} \mathrm{MgSO}_{4}$. $7 \mathrm{H}_{2} \mathrm{O}, 0.5 \mathrm{mM} \mathrm{NaHCO}{ }_{3}, 0.2 \mathrm{mM} \mathrm{CaCl} \cdot \mathrm{H}_{2} \mathrm{O}, 0.2 \mathrm{mM}$ $\mathrm{KH}_{2} \mathrm{PO}_{4}, 0.4 \mathrm{mM} \mathrm{NH} \mathrm{m}_{4} \mathrm{Cl}$, and $0.6 \mathrm{mM}$ sodium citrate $(\mathrm{pH}$ 7.5) was prepared based on the reported protocol. ${ }^{43}$

Bacterial attachment was evaluated by placing circular membrane coupons with the diameter of $1.6 \mathrm{~cm}$ were placed in sterile plastic tubes with $5 \mathrm{~mL}$ of $P$. aeruginosa suspension at $37{ }^{\circ} \mathrm{C}$ for $1 \mathrm{~h}$. Biofouled membrane coupons were then collected and gently rinsed using sterile synthetic wastewater to remove unattached cells. Live and dead cells were stained with $3.34 \mu \mathrm{M}$ SYTO9 and $20 \mu \mathrm{M}$ PI solution, respectively, for 30 min in the dark. The membranes were then rinsed three times with sterile synthetic wastewater to remove unbound stains. The fluorescence images of the stained membrane coupons were collected using a confocal laser scanning microscopy (CLSM, Fluo View FV1000, Olympus). SYTO9 was excited with an argon laser at $488 \mathrm{~nm}$, and PI was excited with a diodepumped solid-state laser at $559 \mathrm{~nm}$. Live cells and dead cells were recognized as green and red spots, respectively. The CLSM images were analyzed using ImageJ Pro software (National Institutes of Health). For quantitative image analysis, three individual samples were measured in a parallel manner, and the results were reported as the average values with the standard deviations.

Antimicrobial Activity Measurements. The antimicrobial activity of Ag NPs-containing TFC FO membranes was assessed using the reported colony-forming unit (CFU) method. ${ }^{38}$ Circular membrane coupons with $1.6 \mathrm{~cm}$ in diameter were cut from the freshly prepared membranes and then incubated with $P$. aeruginosa suspensions $\left(10^{8} \mathrm{CFU}\right.$ $\mathrm{mL}^{-1}$ ) in the mid-exponential growth phase for $5 \mathrm{~h}$ at $37^{\circ} \mathrm{C}$.
After gentle rinsing with sterile physiological saline solution

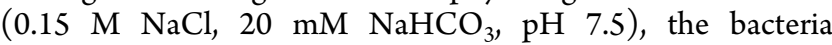
attached on the membrane coupons were removed through 7 min bath-sonication in $10 \mathrm{~mL}$ of sterile physiological saline solution. The bacterial suspensions were serially diluted 100 times, and $100 \mu \mathrm{L}$ of the dilution was taken to spread onto an LB agar plate. Then bacteria colonies were counted after $24 \mathrm{~h}$ of incubation at $37{ }^{\circ} \mathrm{C}$. The antibacterial efficiency $E_{\mathrm{b}}$ was calculated by the following equation:

$$
E_{\mathrm{b}}=\frac{\left(N_{\mathrm{p}}-N_{\mathrm{m}}\right)}{N_{\mathrm{p}}} \times 100 \%
$$

where $N_{\mathrm{p}}$ and $N_{\mathrm{m}}$ are the numbers of colonies formed on pristine membranes and modified membranes, respectively.

Dynamic Biofouling Experiments and Biofilm Characterization. The dynamic biofouling measurements were carried out in a lab-scale FO cross-flow setup with the effective surface area of $8 \mathrm{~cm}^{2}$ reported in our previous work. ${ }^{41}$ The FO setup was stabilized using DI water as both feed solution and draw solution to obtain the water flux of 0 for $20 \mathrm{~min}$. The synthetic wastewater stock and $\mathrm{NaCl}$ stock was added into the feed solution side and draw solution side separately to achieve a permeate water flux of $20 \mathrm{~L} \mathrm{~m}^{-2} \mathrm{~h}^{-1}$. P. aeruginosa suspension was then added into the feed solution side to reach a bacterial concentration of $6.0 \times 10^{7} \mathrm{CFU} \mathrm{L}^{-1}$. The volumes of feed and draw solutions were $2 \mathrm{~L}$ for all of the experiments. The $\mathrm{pH}$ of feed solution was adjusted to $\mathrm{pH} 7.5$ during the dynamic biofouling experiment. The cross-flow rates of both feed and draw solutions were maintained at $0.5 \mathrm{~L} \min ^{-1}$ and the temperatures of solutions were kept at $25 \pm 0.5{ }^{\circ} \mathrm{C}$. The water flux was continuously monitored by measuring the weight increment of the draw solution using an electronic balance (ME3002, Mettler, Switzerland). The FO filtration tests were operated under both FO and PRO modes, respectively.

Biofilms were analyzed upon staining using CLSM. Briefly, 1 $\mathrm{cm}^{2}$ of biofouled membrane coupon was cut from the tested membranes and then stained with SYTO9, PI, and $50 \mu \mathrm{M}$ Con A for $30 \mathrm{~min}$ in dark to label live cells, dead cells, and extracellular polymeric substance (EPS), respectively. A Zstack scanning with a slice thickness of $2 \mu \mathrm{m}$ was performed to collect the images of biofilm. The three-dimensional structure of biofilm was reconstructed using the FV1000 Viewer software (Olympus). Con A was excited with a diode-pumped solid-state laser at $633 \mathrm{~nm}$.

Stability of Ag NPs on TFC Membrane. To evaluate the stability of Ag NPs on TFC membrane, the membranes coupons were tested for $24 \mathrm{~h}$ in the FO cross-flow system and then taken out from the $\mathrm{FO}$ cell to cut as $1 \mathrm{~cm} \times 1 \mathrm{~cm}$ membrane coupons. Their antimicrobial activity was determined using the CFU method following the above-mentioned protocol. The residual amount of $\mathrm{Ag} \mathrm{NPs}$ on the tested membranes was quantified using an inductively coupled plasma mass spectrometer (ICP-MS). Membrane coupons were immersed in $10 \mathrm{~mL}$ of $3.5 \% \mathrm{HNO}_{3}$ solution for $48 \mathrm{~h}$ to completely dissolve Ag NPs in the membrane. The concentrations of silver ions in the solutions were measured using ICP-MS (ELAN DRC II, PerkinElmer).

Total Organic Carbon and Total Protein Analysis. The total organic carbon (TOC) measurement was performed according to the reported protocol, ${ }^{19}$ and membrane coupons were cut $(1 \mathrm{~cm} \times 1 \mathrm{~cm})$ from the center of biofouled membranes and placed into sterile glass bottles with $20 \mathrm{~mL}$ of 

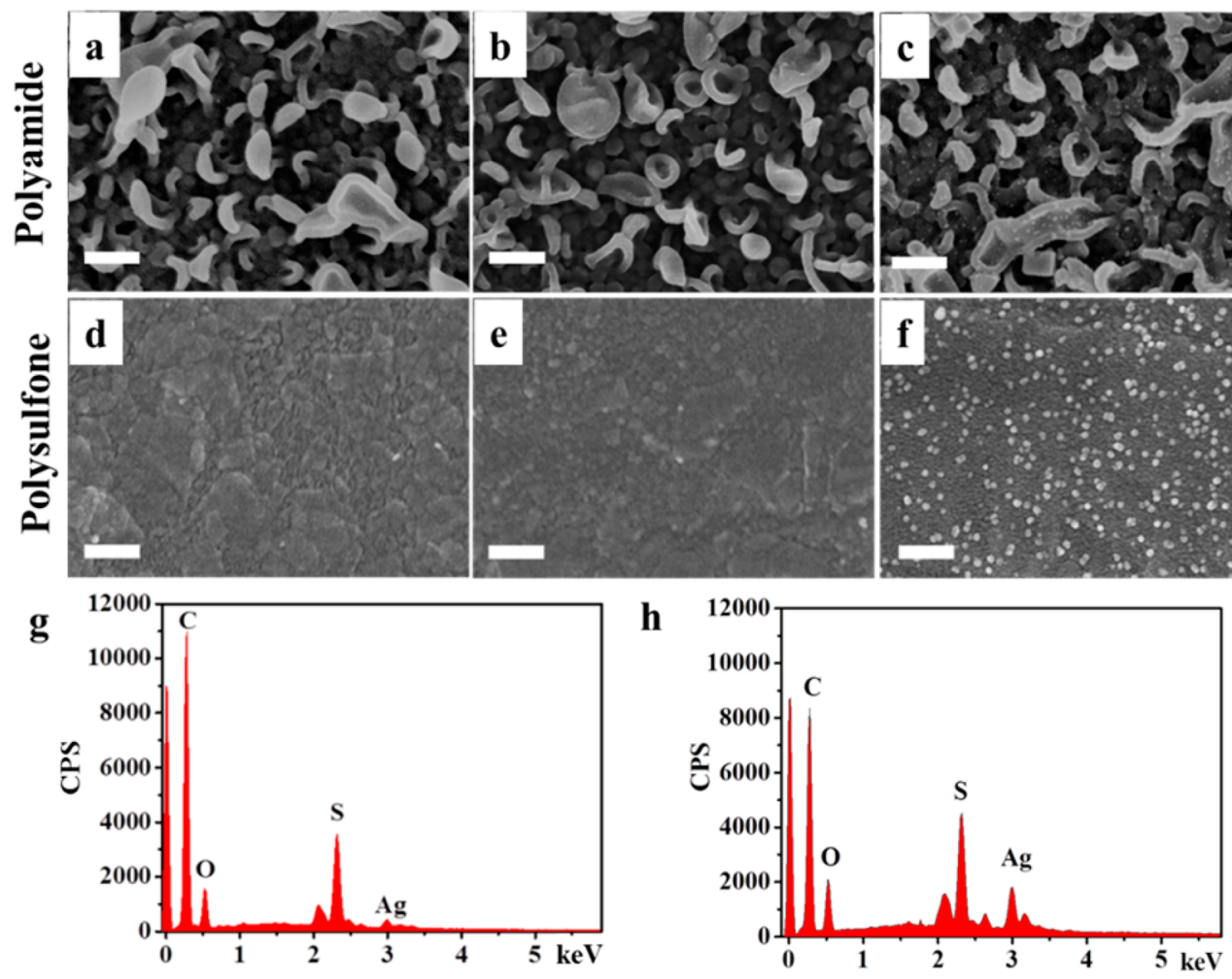

Figure 1. Representative SEM micrographs of the top polyamide surface and the bottom polysulfone surface from (a, d) pristine, (b, e) PDAcoated, and (c, f) Ag-NP-generated TFC membranes and corresponding EDX spectra of Ag-NP-generated (g) polyamide surface and (h) polysulfone surface. Scale bars in SEM are $200 \mathrm{~nm}$.

hydrogen chloride aqueous solution $(20 \mathrm{mM})$. The biofilm was removed from the membrane coupons through $60 \mathrm{~s}$ of sonication using a probe sonicator (JY92-IIDN, Scientz biotechnology Ning Bo Co., Ltd., China), and TOC in the solution was quantified using a TOC analyzer (TOC-VCPH, Shimadzu, Japan) according to the operation manual of manufacture. The TOC of biofilm was reported as the average value from three parallel samples and the standard deviation as the error bar after normalization by the surface area of membrane coupons.

Total protein biomass was quantified using the reported BCA method. ${ }^{44,45}$ Biofouled membrane coupons $(1 \mathrm{~cm} \times 1$ $\mathrm{cm}$ ) were placed into $2 \mathrm{~mL}$ Eppendorf tubes with $1 \mathrm{~mL}$ of PBS buffer and then sonicated for $7 \mathrm{~min}$ to break bacteria cells. After centrifugation at $13000 \mathrm{rpm}$ for $10 \mathrm{~min}$, the protein in the supernatant solution was quantified using a BCA protein assay kit (Institute of Biological Engineering Co., Ltd., China) according to the operation manual of manufacture. The total protein biomass of biofilm was reported as the average value from three parallel samples and the standard deviation as the error bar after normalization by the surface area of membrane coupons.

\section{RESULTS AND DISCUSSION}

PDA Coating and Ag NPs Generation and Increase of Surface Hydrophilicity without Affecting the Transport Properties of TFC FO Membranes. Our previous work had demonstrated that Ag NPs were in situ generated on both surfaces of TFC FO membranes, including the top polyamide surface and the bottom PET-strengthened polysulfone surface via mussel-inspired dopamine chemistry. ${ }^{33}$ Here, we further investigated the optimized experimental conditions of in situ
Ag NPs generation on both surfaces of TFC FO membranes. SEM analysis indicates that the PDA coating did not present morphology change between the PDA coated membranes (Figure $1 \mathrm{~b}, \mathrm{e}$ ) and pristine membranes (Figure 1a,d) on the polyamide surface and the polysulfone surface. Ag NPs were densely populated and uniformly distributed on characteristic ridge-and-valley polyamide surface (Figures $1 \mathrm{c}$ and S1) as well as a smooth polysulfone surface (Figure 1f). Interestingly, the Ag NPs generated on the polysulfone surface was $28.1 \pm 4.7$ $\mathrm{nm}$ in diameter, which is bigger than $10.6 \pm 2.5 \mathrm{~nm}$ in diameter of Ag NPs formed on the polyamide surface. This change in Ag NPs size is likely due to the different surface morphologies and properties of polysulfone surface and polyamide surface, which affects the growth of PDA coating and thus tailors the nucleation and growth of Ag NPs. ${ }^{33,46}$ In addition, the Ag NPs generated on the polysulfone surface presented stronger silver signals than that on the polyamide surface, indicating the greater silver mass deposited on the polysulfone surface.

PDA imparts its hydrophilicity to membrane surfaces once its coating forms because it decreased the water-contact angles of both the polyamide layer and PSf support from $70.8 \pm 4.6$ and $125.4 \pm 3.6^{\circ}$ to $43.8 \pm 5.7$ and $79.1 \pm 8.7^{\circ}$, respectively, as shown in Table S1. The Ag NP generation further decreased the water contact angles of polyamide layer and PSf support to $37.9 \pm 3.8^{\circ}$ and $75.1 \pm 8.6^{\circ}$, respectively, and, thus, improved the membrane surface hydrophilicity because of the hydrophilic nature of Ag NPs and the improved surface roughness of Ag NPs modified membranes. ${ }^{47,48}$ Noticeably, the pristine PSf support surface showed a quite-high water-contact angle due to the hydrophobic PET nonwoven fabric and polysulfone on the on the back surface of the TFC FO membrane (Figure S2). 
a
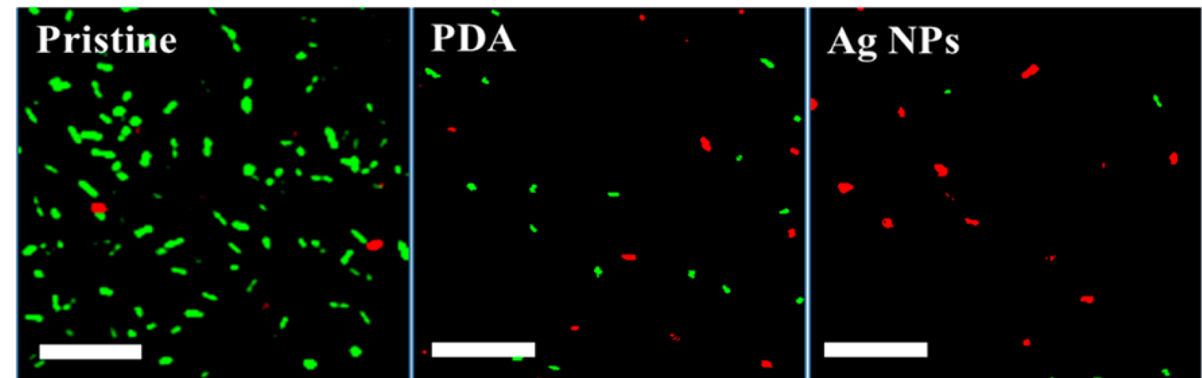

b
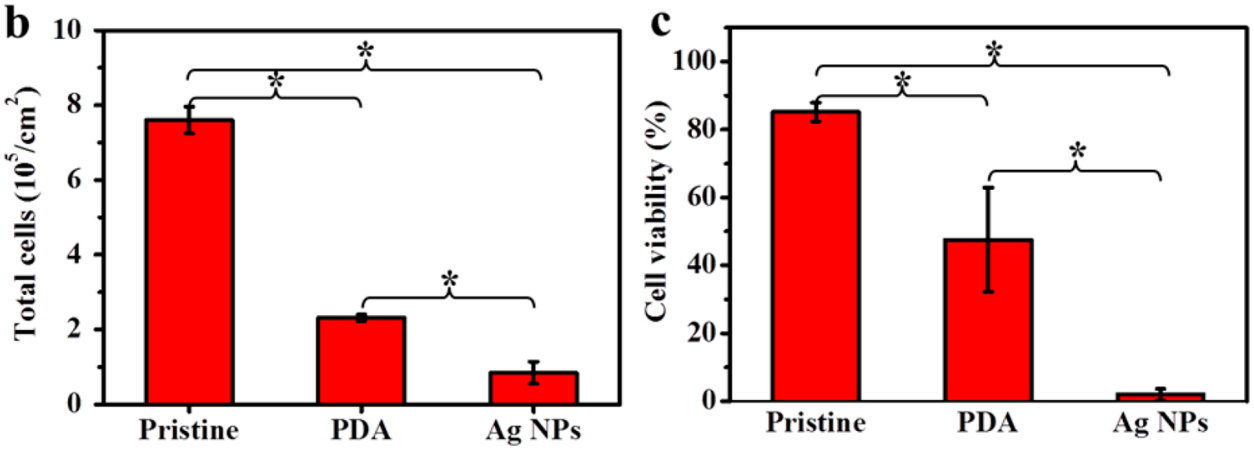

Figure 2. Static anti-adhesion properties of TFC FO membranes. (a) Representative CLSM images of P. aeruginosa cells attached on the pristine, PDA-coated, and Ag-NP-generated polysulfone surfaces of TFC FO membranes and (b) total number and (c) viability of P. aeruginosa cell attached on membrane surfaces. Results are presented as an average of at least three different membrane samples, and error bars are given as standard deviations. Bacterial cells were stained with SYTO9 (green) and PI (red) for live and dead cells, respectively. Scale bars in CLSM are 10 $\mu \mathrm{m}$. Asterisks indicate statistical significance determined by a Student $t$ test $(p$ value of $<0.05)$.

Furthermore, the $\zeta$-potential measurements shown in Table S1 found that the PDA coating and Ag NPs generation did not affect the $\zeta$ potentials of TFC FO membranes and, therefore, did not change their surface charges.

The impacts of PDA coating and Ag NPs on the transport properties of TFC FO membrane were investigated to optimize surface modification conditions. PDA coating had no major effect on membrane separation performance within 1 $\mathrm{h}$ of modification due to the formation of thin PDA layer. ${ }^{33,39}$ Under the condition of $1 \mathrm{~h}$ of PDA coating, Ag NP generation had a negligible effect on membrane water fluxes within $2 \mathrm{~h}$ of modification and then resulted in $10 \%$ flux reduction with increasing the incubation time to $8 \mathrm{~h}$ (Figure S3a). In addition, the reverse salt flux of the TFC FO membrane was not affected by Ag NPs generation. Under the optimized conditions of $1 \mathrm{~h}$ of PDA coating and $2 \mathrm{~h}$ of $\mathrm{AgNO}_{3}$ immersion time, the PDAcoated and Ag-NP-generated TFC FO membranes were prepared, and their intrinsic transport parameters were measured following the reported protocol. ${ }^{49}$ The results shown in Figure S3b illustrate that the PDA coating and Ag NPs generation had no significant impact on membrane transport properties.

PDA Coating and Ag NPs Imparting of Membrane Anti-Adhesive and Antimicrobial Properties. Bacteria attachment on membrane surface is a critical step of biofilm formation and development during biofouling. ${ }^{17}$ Thus, the investigation of bacterial adhesion on membrane surface is very conducive to understanding the mechanism of biofouling control. As shown in Figure 2a, the total bacterial cells attached on the membrane surface decreased by $70 \%$ from $7.6 \times 10^{5}$ cells per square centimeter to $2.3 \times 10^{5}$ cells per square centimeter for the pristine and PDA-coated polysulfone support, respectively (Figure $2 \mathrm{~b}$ ). Concurrently, the cell viability on the PDA-coated membrane surface was $47 \%$, lower than $86 \%$ of cell viability on the pristine membrane surface (Figure 2c). Moreover, Ag NP generation further decreased the total attached cells by $65 \%$ to $0.8 \times 10^{5}$ cells per square centimeter and reduced the cell viability by $96 \%$ compared to the PDA-coated membrane (Figure 2b,c). On the polyamide surface of TFC FO membranes, PDA coating has reduced the number of attached cells by $85 \%$ and deactivated $30 \%$ cells compared to the pristine membrane (Figure S4). Ag NP generation further decreased the cell viability by $94 \%$ compared to the PDA-coated membrane (Figure S4). It can conclude that the PDA coating exhibits both the anti-adhesive property and the antibacterial performance. The anti-adhesive effect of PDA coating has been extensively reported due to the formation of a hydration layer from the enhanced surface hydrophilicity mitigating the adsorption of contaminants. ${ }^{35}$ The bacterial biocide effect of PDA coating is related to the protonated amine groups, which can cause bacteria lysis and deactivate bacteria by contacting the cell wall of bacteria. ${ }^{50}$ Even though the surface charge of membrane surface is negative at $\mathrm{pH} 7.5$, the protonation degree of amine groups in PDA was reported as $94 \% .^{35,51}$ The smaller viability of bacteria on PDA-coated membranes is not only due to the antibacterial properties of PDA but also due to the less number of bacteria attached on PDA coted surfaces. Above all, these results illustrate that the PDA coating could effectively prevent the bacterial adhesion on membrane surface, and the Ag NPs generation could successfully deactivate the attached bacteria under static conditions.

Membrane Biofouling Mitigation by Ag NPs during Forward-Osmosis Operation. Membrane biofouling is a dynamic process involving the continuous adsorption of microorganisms to membrane surfaces and their growth and development into biofilm. ${ }^{52}$ The anti-biofouling performances of the modified TFC FO membranes were also investigated in 

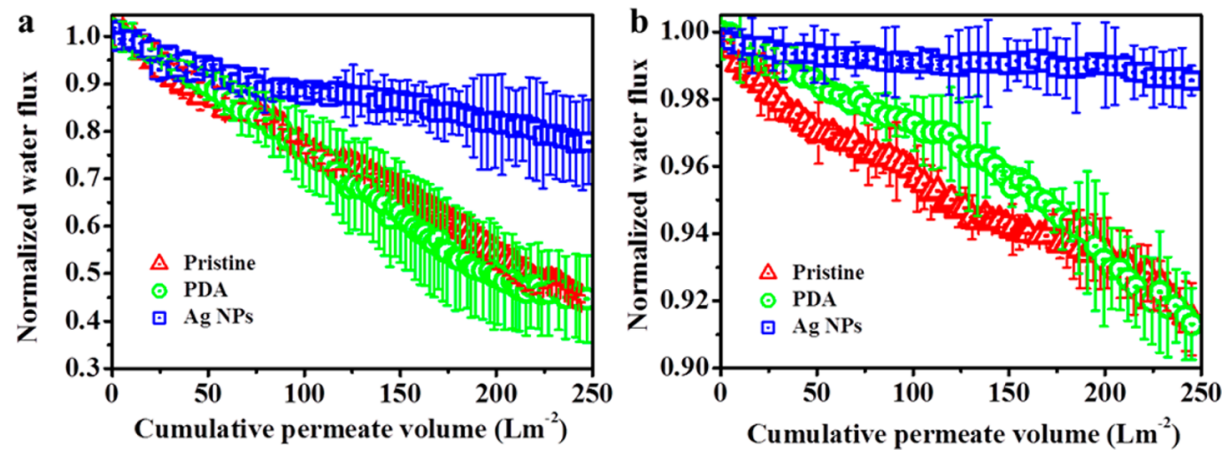

Figure 3. Dynamic biofouling performance of TFC FO membranes under the PRO and FO operation modes. (a) Normalized water flux of TFC FO membranes as a function of cumulative permeate volume during the $P$. aeruginosa biofilm development on polysulfone support under the PRO operation mode and (b) normalized water flux of TFC FO membranes as a function of cumulative permeate volume during the $P$. aeruginosa biofilm development on polyamide surfaces under the FO operation mode. The cumulative permeate volume of $250 \mathrm{~L} \mathrm{~m}^{-2}$ corresponds to 21 to 24 $\mathrm{h}$ of biofouling experiment duration depending on the membrane water flux.

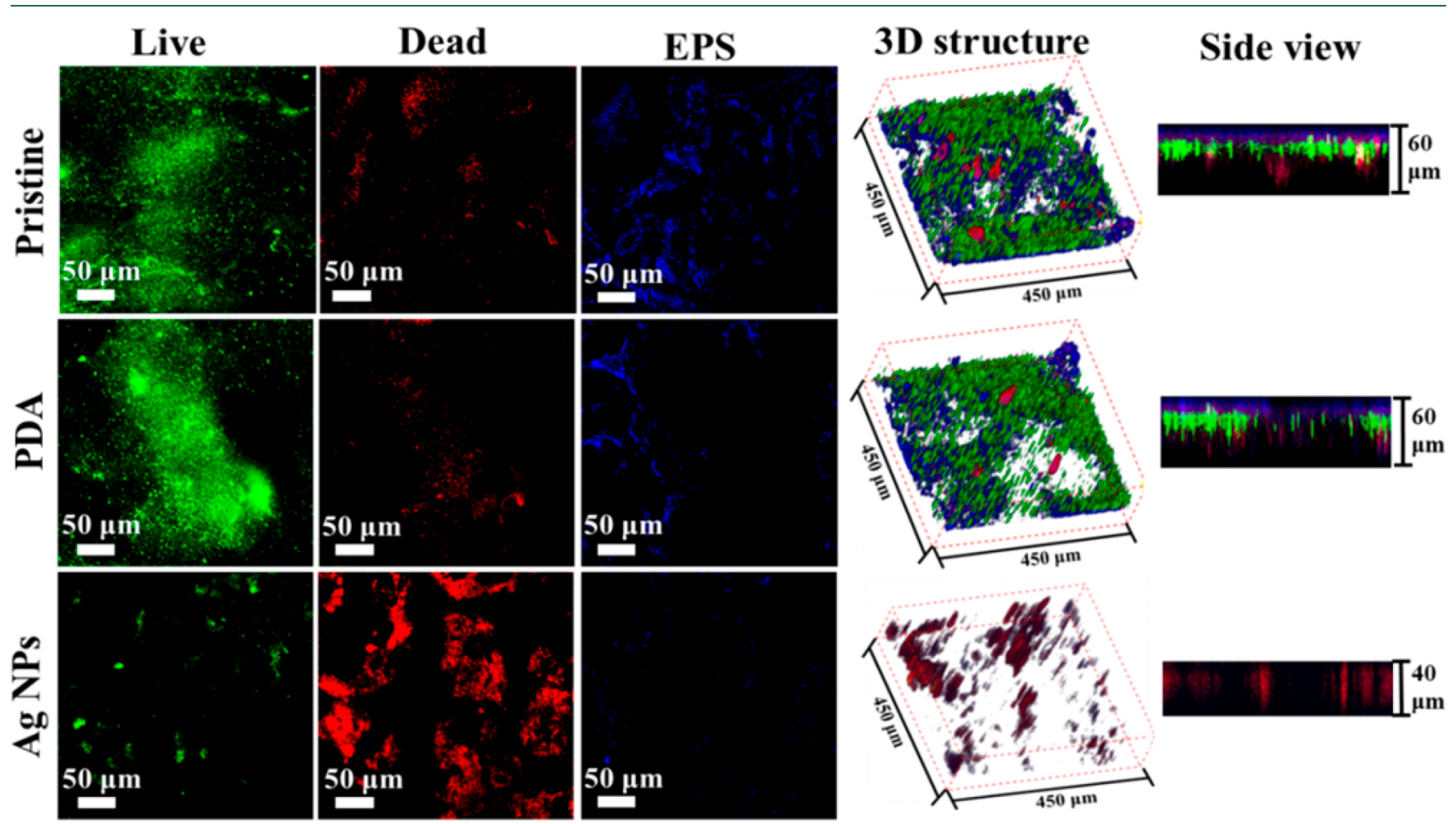

Figure 4. Representative top-view, 3D structure, and side-view images of the biofilms formed on the polysulfone support of TFC FO membranes. Biofilms were stained with SYTO9 (green), PI (red), and Con A (blue) for live cells, dead cells, and EPS, respectively.

the dynamic biofouling experiments. Because both the polyamide surface and polysulfone support were modified with PDA and Ag NPs, it provides a unique opportunity to evaluate their anti-biofouling properties running on both the FO mode and the PRO mode. Figure 3a shows that a gradual yet continuous decline in permeate water flux was observed for all membranes under the PRO mode due to biofouling. Permeate water flux for the pristine membrane declined by $56.4 \%$ during $24 \mathrm{~h}$ of operation. Under the same conditions, permeate water flux declined for the PDA-coated membranes and the Ag NPs generated membranes by $56.6 \%$ and $21.9 \%$, respectively. Conducting the biofouling experiment under the FO mode resulted in a $8.5 \%$ decline in the permeate water flux of the pristine membrane (Figure 3b). The PDA-coated membranes exhibited similar water flux decline behavior as the pristine membrane during the biofouling experiments.
Importantly, the Ag-NP-generated membranes exhibited only $0.5 \%$ permeate water flux decline and presented great biofouling mitigation performances. Even Ag-NP-generated TFC membranes have also PDA coating, and all of the above results illustrate that only Ag NPs rather than PDA coating can substantially suppress the membrane permeate water flux decline and mitigate membrane biofouling.

Moreover, the results shown in Figure 3a,b also indicate that the same TFC FO membrane suffered more-severe water flux decline when running in the PRO mode than in the FO mode. This is because the bottom support layer of TFC membranes has numerous large pores that is more prone to be clogged with foulants inside. ${ }^{13}$ In contrast, bacteria can deposit and develop into biofilm only on the surface of top selective layer due to the dense structure of polyamide. Moreover, the TFC FO membrane suffers more-severe fouling-enhanced internal 

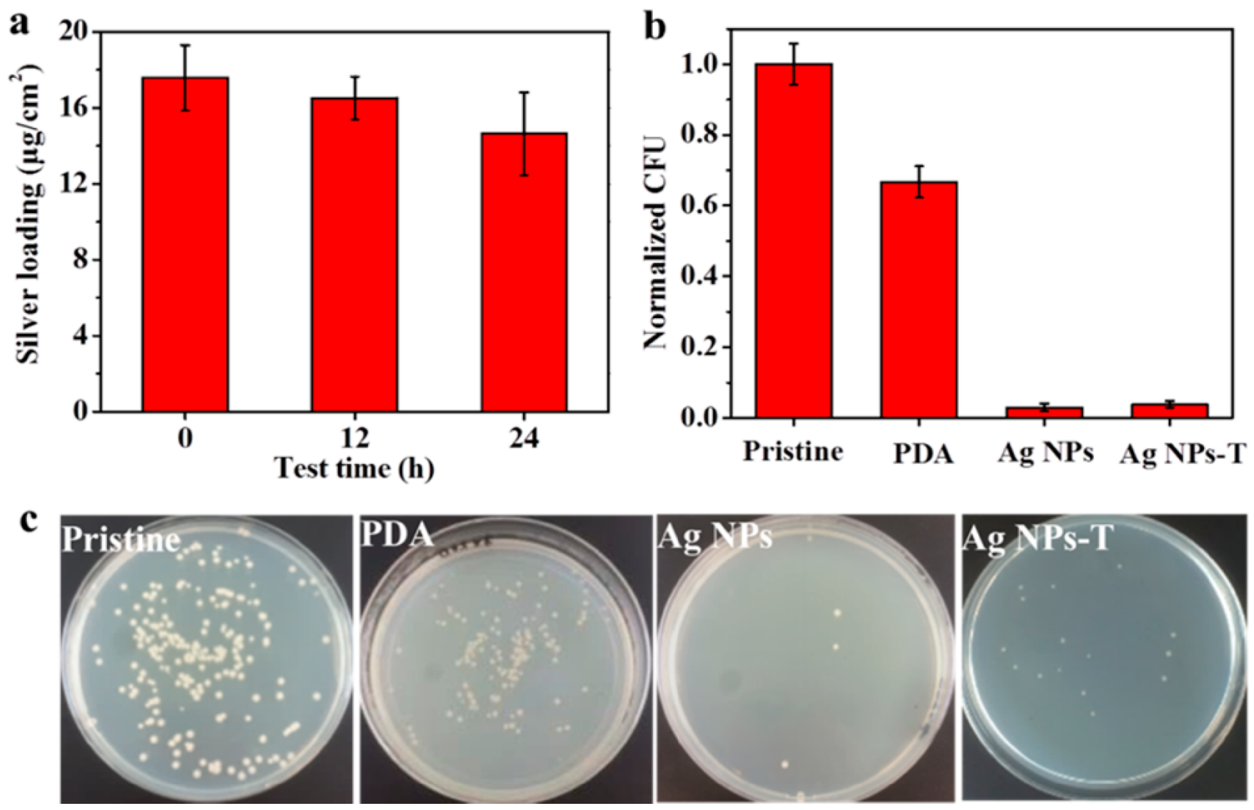

Figure 5. Stability and activity of Ag NPs on TFC FO membrane. (a) Residual silver amount on TFC FO membranes after the cross-flow test in the FO system; (b) antimicrobial efficiency of pristine membranes, PDA-coated membranes, Ag-NP-generated membranes, and Ag-NP-generated membranes after $24 \mathrm{~h}$ of cross-flow running in the FO system (Ag NPs-T); and (c) the corresponding bacterial culture plate photographs.

concentration polarization in the PRO mode than in the FO mode. $^{28}$

Interestingly, PDA was able to slow the membrane flux decline in the FO mode when the permeate volume was lower than $175 \mathrm{~L} \mathrm{~m}^{-2}$ (Figure $3 \mathrm{~b}$ ) compared to the pristine membrane, which indicates that the passive moieties have shown some effective anti-biofouling performances. This is because that the experimental conditions favored foulantmembrane interaction, and thus, the passive moieties on membrane surface mitigated the biofouling during the initial biofouling stage. With the progress of biofouling and the development of biofilm, PDA lost its antifouling properties because of its inability to inhibit biofilm development because the experimental conditions favored the foulant-foulant interaction and the PDA coating had no impact on the deposition of bacteria when the membrane surface was fully covered and masked by biofilm. The transition from the stage of the foulant-membrane interaction to that of foulantfoulant dominant interaction was not observed in the PRO mode, which is likely due to the severe fouling conditions in the PRO mode. In addition, a similar outcome was found for other ultrafiltration and nanofiltration membranes modified with hydrophilic polymers such as PDA, poly(ethylene glycol), or heparin; the static anti-adhesion properties of hydrophilic membrane surfaces did not contribute to the enhancement of anti-biofouling performances. ${ }^{30,53}$

Ag NPs Impairing of Biofilm Growth on TFC FO Membrane Surfaces. To investigate the biofouling mitigation mechanisms of modified TFC membranes, the formed biofilms were analyzed using CLSM. The results shown in Figure 4 indicate that dead cells were more-abundant, while live cells and EPS were less-abundant on the Ag-NP-generated membranes than on the PDA -coated and pristine TFC membranes. Moreover, the top layer of the biofilm formed on the Ag-NP-generated membrane was mainly composed of dead cells, indicating that silver ions leached and diffused to the upper layers of biofilm and broke cell integrity. ${ }^{54}$ The image analysis of 3D and the side view of the biofilm further confirms that the biofilm formed on the Ag-NP-generated membrane was thinner and mainly composed of more dead cells with fewer live cells and EPS than biofilms formed on both the PDA-coated and the pristine membrane surfaces. These results indicate that the antimicrobial activity rendered by Ag NPs inhibited the development of biofilm on membrane surfaces.

Quantitative analysis was performed to investigate the biomass of biofilms. Less TOC and total protein biomass were found on the Ag NPs generated polysulfone support than that on the PDA coated and pristine membranes (Figure S5). In addition, the PDA coating decreased the TOC content by $29 \%$ without affecting the protein biomass on membrane surfaces compared to the pristine surface, which may be due to the suppressed adhesion of organic compounds in feed solution without affecting bacterial growth. Above all, it can be concluded that the antimicrobial activity rendered by $\mathrm{Ag}$ NPs inhibits the development of biofilm on membrane surfaces and thus mitigates the membrane biofouling. Moreover, the anti-adhesion properties of hydrophilic membrane surfaces with PDA coating can neither prevent biofilm growth nor mitigate membrane biofouling. However, the anti-adhesion properties of PDA coating has a potential benefit of the efficient removal of biofilm from the modified membrane surfaces during the backwash cleaning procedure, which need a future comprehensive study.

Ag NP Stability and Activity on TFC FO Membrane. The stability and activity of Ag NPs on TFC FO membrane was investigated during the dynamic cross-flow operation. We quantified the silver content on membranes with ICP-MS and found that the silver content decreased by $6 \%$ after $12 \mathrm{~h}$ of operation and further decreased by $17 \%$ after $24 \mathrm{~h}$ of operation (Figure 5a). The morphologies of Ag NPs on polysulfone surface before and after $24 \mathrm{~h}$ of operation were observed using an SEM. The average diameter of Ag NPs decreases from 28.1 \pm 4.7 to $21.9 \pm 5.8 \mathrm{~nm}$, and the number of $\mathrm{Ag}$ NPs decreases from $141 \pm 19$ to $100 \pm 26$ per square micrometer (Figure 
S6). Therefore, the silver leaching comes from both losing particles and the dissolution of the Ag NPs. The antimicrobial efficiency of TFC FO membrane was investigated using the CFU counting method. Results shown in Figure 5b,c present that the numbers of attached live $P$. aeruginosa decreased by $33.3 \pm 4.2 \%$ and $97.0 \pm 2.4 \%$ for the PDA-coated and the Ag$\mathrm{NP}$-generated membranes compared to the pristine membrane, agreeing with the results of CLSM image analysis in the static condition. The Ag NPs generated membranes after $24 \mathrm{~h}$ of cross-flow running in the FO system (Ag NPs-T) still exhibited great antimicrobial efficiency of $96.1 \%$, similar to that of the fresh Ag-NP-generated membrane. Therefore, the Ag NPs are stable and active on TFC FO membrane and hold great potential for sustainable antibacterial performance.

Implications for Biofouling Mitigation of TFC FO Membrane. In this study, we fabricated TFC FO membranes modified with both PDA as the passive moiety and Ag NPs as the active moiety and investigated the performances and mechanisms of their biofouling mitigation in the $\mathrm{FO}$ process. The PDA coating on TFC FO membranes exhibits passive anti-adhesive properties owing to hydrophilicity in static conditions but shows little effect on biofouling mitigation in the dynamic cross-flow conditions due to its inability to inactivate bacteria growth. The Ag NPs present active antibacterial properties in both the static and the dynamic conditions. The biofilm analysis illustrates that the mechanism of biofouling mitigation by Ag NPs is the prevention of biofilm growth and the reduction of microbial biomass through the inactivation of the attached bacteria. In addition, TFC FO membranes suffered more-severe water flux decline from biofouling when running in the PRO mode than in the FO mode. More importantly, Ag NPs show good stability and activity on the TFC FO membrane surfaces during $24 \mathrm{~h}$ of cross-flow operation. These findings provide new insights on the effective surface engineering of FO membranes with active moieties for efficient biofouling mitigation. Future investigations should focus on the long-term anti-biofouling properties of TFC FO membranes with the passive and active moieties when coupled with cleaning strategies to treat complex water systems.

\section{ASSOCIATED CONTENT}

\section{(S) Supporting Information}

The Supporting Information is available free of charge on the ACS Publications website at DOI: 10.1021/acs.est.7b06382.

Figures showing SEM images, the impact of Ag NP generation, a static anti-adhesion study, and TOC and total protein biomass analysis of the biofilms. A table showing the water contact angle and $\zeta$ potential of the TFC FO membranes. (PDF)

\section{AUTHOR INFORMATION}

\section{Corresponding Author}

*Tel: +86-22-83955129; e-mail: yunxiahu@yic.ac.cn. ORCID $\odot$

Yunxia Hu: 0000-0003-4710-6542

Zhongyun Liu: 0000-0002-9078-0404

Xiaochan An: 0000-0002-4765-3178

Edo Bar-Zeev: 0000-0001-6575-2068

Notes

The authors declare no competing financial interest.

\section{ACKNOWLEDGMENTS}

The authors gratefully acknowledge the funding support from National Natural Science Foundation of China (nos. 21476249 and 51708408), the Chang-Jiang Scholars and Innovative Research Team in the University of Ministry of Education, China (no. IRT-17R80), Program for Innovative Research Team in University of Tianjin (no. TD13-5044), and the Science and Technology Plans of Tianjin (no. 17PTSYJC00060).

\section{REFERENCES}

(1) Vörösmarty, C. J.; Green, P.; Salisbury, J.; Lammers, R. B. Global water resources: vulnerability from climate change and population growth. Science 2000, 289 (5477), 284-288.

(2) Shannon, M. A.; Bohn, P. W.; Elimelech, M.; Georgiadis, J. G.; Mariñas, B. J.; Mayes, A. M. Science and technology for water purification in the coming decades. Nature 2008, 452 (7185), 301310.

(3) Elimelech, M.; Phillip, W. A. The future of seawater desalination: energy, technology, and the environment. Science 2011, 333 (6043), 712-717.

(4) Shaffer, D. L.; Werber, J. R.; Jaramillo, H.; Lin, S.; Elimelech, M. Forward osmosis: Where are we now? Desalination 2015, 356, 271284.

(5) Lutchmiah, K.; Verliefde, A. R.; Roest, K.; Rietveld, L. C.; Cornelissen, E. R. Forward osmosis for application in wastewater treatment: a review. Water Res. 2014, 58 (3), 179-197.

(6) Cornelissen, E. R.; Harmsen, D.; de Korte, K. F.; Ruiken, C. J.; Qin, J. J.; Oo, H.; Wessels, L. P. Membrane fouling and process performance of forward osmosis membranes on activated sludge. $J$. Membr. Sci. 2008, 319 (1-2), 158-168.

(7) Wintgens, T.; Melin, T.; Schäfer, A.; Khan, S.; Muston, M.; Bixio, D.; Thoeye, C. The role of membrane processes in municipal wastewater reclamation and reuse. Desalination 2005, 178 (1), 1-11.

(8) Han, G.; Zhang, S.; Li, X.; Chung, T. S. High performance thin film composite pressure retarded osmosis (PRO) membranes for renewable salinity-gradient energy generation. J. Membr. Sci. 2013, 440 (8), 108-121.

(9) Logan, B. E.; Elimelech, M. Membrane-based processes for sustainable power generation using water. Nature 2012, 488 (7411), 313-319.

(10) Yip, N. Y.; Tiraferri, A.; Phillip, W. A.; Schiffman, J. D.; Elimelech, M. High performance thin-film composite forward osmosis membrane. Environ. Sci. Technol. 2010, 44 (10), 3812-3818.

(11) Han, G.; Zhao, B.; Fu, F.; Chung, T. S.; Weber, M.; Staudt, C.; Maletzko, C. High performance thin-film composite membranes with mesh-reinforced hydrophilic sulfonated polyphenylenesulfone (sPPSU) substrates for osmotically driven processes. J. Membr. Sci. 2016, 502, 84-93.

(12) Ren, J.; Mccutcheon, J. R. A new commercial thin film composite membrane for forward osmosis. Desalination 2014, 343 (12), 187-193.

(13) Barzeev, E.; Perreault, F.; Straub, A. P.; Elimelech, M. Impaired performance of pressure-retarded osmosis due to irreversible biofouling. Environ. Sci. Technol. 2015, 49 (21), 13050-13058.

(14) Yoon, H.; Baek, Y.; Yu, J.; Yoon, J. Biofouling occurrence process and its control in the forward osmosis. Desalination 2013, 325 (20), 30-36.

(15) Lee, S.; Boo, C.; Elimelech, M.; Hong, S. Comparison of fouling behavior in forward osmosis (FO) and reverse osmosis (RO). J. Membr. Sci. 2010, 365 (1), 34-39.

(16) Zhang, R.; Liu, Y.; He, M.; Su, Y.; Zhao, X.; Elimelech, M.; Jiang, Z. Antifouling membranes for sustainable water purification: strategies and mechanisms. Chem. Soc. Rev. 2016, 45 (21), 58885924. 
(17) Mansouri, J.; Harrisson, S.; Chen, V. Strategies for controlling biofouling in membrane filtration systems: challenges and opportunities. J. Mater. Chem. 2010, 20 (22), 4567-4586.

(18) Banerjee, I.; Pangule, R. C.; Kane, R. S. Antifouling coatings: recent developments in the design of surfaces that prevent fouling by proteins, bacteria, and marine organisms. Adv. Mater. 2011, 23 (6), 690-718.

(19) Perreault, F.; Jaramillo, H.; Xie, M.; Ude, M.; Elimelech, M.; Nghiem, L. D. Biofouling mitigation in forward osmosis using graphene oxide functionalized thin-film composite membranes. Environ. Sci. Technol. 2016, 50 (11), 5840-5848.

(20) Rahaman, M. S.; Thérienaubin, H.; Bensasson, M.; Ober, C. K.; Nielsen, M.; Elimelech, M. Control of biofouling on reverse osmosis polyamide membranes modified with biocidal nanoparticles and antifouling polymer brushes. J. Mater. Chem. B 2014, 2 (12), 17241732.

(21) Li, X.; Cai, T.; Chung, T. S. Anti-fouling behavior of hyperbranched polyglycerol-grafted poly(ether sulfone) hollow fiber membranes for osmotic power generation. Environ. Sci. Technol. 2014, 48 (16), 9898-9907.

(22) Kochkodan, V.; Hilal, N. A comprehensive review on surface modified polymer membranes for biofouling mitigation. Desalination 2015, 356, 187-207.

(23) Zhu, L. J.; Zhu, L. P.; Zhao, Y. F.; Zhu, B. K.; Xu, Y. Y. Antifouling and anti-bacterial polyethersulfone membranes quaternized from the additive of poly(2-dimethylamino ethyl methacrylate) grafted SiO2 nanoparticles. J. Mater. Chem. A 2014, 2 (37), 15566.

(24) Tang, C. Y.; Kwon, Y. N.; Leckie, J. O. The role of foulantfoulant electrostatic interaction on limiting flux for $\mathrm{RO}$ and NF membranes during humic acid fouling-Theoretical basis, experimental evidence, and AFM interaction force measurement. J. Membr. Sci. 2009, 326 (2), 526-532.

(25) Liu, X.; Foo, L. X.; Li, Y.; Lee, J. Y.; Cao, B.; Tang, C. Y. Fabrication and characterization of nanocomposite pressure retarded osmosis (PRO) membranes with excellent anti-biofouling property and enhanced water permeability. Desalination 2016, 389, 137-148.

(26) Wang, J.; Wang, Y.; Zhang, Y.; Uliana, A. A.; Zhu, J.; Liu, J.; van der Bruggen, B. Zeolitic imidazolate framework/graphene oxide hybrid nanosheets functionalized thin film nanocomposite membrane for enhanced antimicrobial performance. ACS Appl. Mater. Interfaces 2016, 8 (38), 25508-25519.

(27) Ben-Sasson, M.; Zodrow, K. R.; Genggeng, Q.; Kang, Y.; Giannelis, E. P.; Elimelech, M. Surface functionalization of thin-film composite membranes with copper nanoparticles for antimicrobial surface properties. Environ. Sci. Technol. 2014, 48 (1), 384-393.

(28) She, Q.; Wang, R.; Fane, A. G.; Tang, C. Y. Membrane fouling in osmotically driven membrane processes: A review. J. Membr. Sci. 2016, 499, 201-233.

(29) Kang, S. T.; Subramani, A.; Hoek, E. M. V.; Deshusses, M. A.; Matsumoto, M. R. Direct observation of biofouling in cross-flow microfiltration: mechanisms of deposition and release. J. Membr. Sci. 2004, 244 (1-2), 151-165.

(30) Liu, C. X.; Zhang, D. R.; He, Y.; Zhao, X. S.; Bai, R. Modification of membrane surface for anti-biofouling performance: Effect of anti-adhesion and anti-bacteria approaches. J. Membr. Sci. 2010, 346 (1), 121-130.

(31) Lee, H.; Dellatore, S. M.; Miller, W. M.; Messersmith, P. B. Mussel-inspired surface chemistry for multifunctional coatings. Science 2007, 318 (5849), 426-430.

(32) Lee, H.; Rho, J.; Messersmith, P. B. Facile conjugation of biomolecules onto surfaces via mussel adhesive protein inspired coatings. Adv. Mater. 2009, 21 (4), 431-43.

(33) Liu, Z.; Hu, Y. Sustainable antibiofouling properties of thin film composite forward osmosis membrane with rechargeable silver nanoparticles loading. ACS Appl. Mater. Interfaces 2016, 8 (33), 21666-21673.

(34) Bernsmann, F.; Ball, V.; Addiego, F.; Ponche, A.; Michel, M.; Gracio, J. J. D. A.; Toniazzo, V.; Ruch, D. Dopamine-melanin film deposition depends on the used oxidant and buffer solution. Langmuir 2011, 27 (6), 2819-2825.

(35) Karkhanechi, H.; Takagi, R.; Matsuyama, H. Biofouling resistance of reverse osmosis membrane modified with polydopamine. Desalination 2014, 336 (1), 87-96.

(36) Mccloskey, B. D.; Park, H. B.; Ju, H.; Rowe, B. W.; Miller, D. J.; Chun, B. J.; Kin, K.; Freeman, B. D. Influence of polydopamine deposition conditions on pure water flux and foulant adhesion resistance of reverse osmosis, ultrafiltration, and microfiltration membranes. Polymer 2010, 51 (15), 3472-3485.

(37) Tang, L.; Livi, K. J. T.; Chen, K. L. Polysulfone membranes modified with bioinspired polydopamine and silver nanoparticles formed in situ to mitigate biofouling. Environ. Sci. Technol. Lett. 2015, 2 (3), 59-65.

(38) Ben-Sasson, M.; Lu, X.; Bar-Zeev, E.; Zodrow, K. R.; Nejati, S.; Qi, G.; Giannelis, E. P.; Elimelech, M. In situ formation of silver nanoparticles on thin-film composite reverse osmosis membranes for biofouling mitigation. Water Res. 2014, 62 (10), 260-270.

(39) Yang, Z.; Wu, Y.; Wang, J.; Cao, B.; Tang, C. Y. In situ reduction of silver by polydopamine: $\mathrm{A}$ novel antimicrobial modification of a thin-film composite polyamide membrane. Environ. Sci. Technol. 2016, 50 (17), 9543-9550.

(40) Liu, Z.; An, X.; Dong, C.; Zheng, S.; Mi, B.; Hu, Y. Modification of thin film composite polyamide membranes with $3 \mathrm{D}$ hyperbranched polyglycerol for simultaneous improvement in their filtration performance and antifouling properties. J. Mater. Chem. A 2017, 5 (44), 23190-23197.

(41) Liu, Z.; An, X.; Liu, C.; Hu, Y.; Qi, L. Surface engineering of thin film composite polyamide membranes with silver nanoparticles through layer-by-layer interfacial polymerization for antibacterial properties. ACS Appl. Mater. Interfaces 2017, 9 (46), 40987-40997.

(42) Liu, Z.; Hu, Y.; Liu, C.; Zhou, Z. Surface-independent one-pot chelating copper ions on filtration membranes for antibacterial properties. Chem. Commun. 2016, 52, 12245-12248.

(43) Xie, M.; Barzeev, E.; Hashmi, S. M.; Nghiem, L. D.; Elimelech, M. Role of reverse divalent cation diffusion in forward osmosis biofouling. Environ. Sci. Technol. 2015, 49 (22), 13222-13229.

(44) Smith, P. K.; Krohn, R. I.; Hermanson, G. T.; Mallia, A. K.; Gartner, F. H.; Provenzano, M. D.; Fujimoto, E. K.; Goeke, N. M.; Olson, B. J.; Klenk, D. C. Measurement of protein using bicinchoninic acid. Anal. Biochem. 1985, 150 (1), 76-85.

(45) Zhao, J.; Shi, Q.; Luan, S.; Song, L.; Yang, H.; Shi, H.; Jin, J.; Li, X.; Yin, J.; Stagnaro, P. Improved biocompatibility and antifouling property of polypropylene non-woven fabric membrane by surface grafting zwitterionic polymer. J. Membr. Sci. 2011, 369 (1-2), 5-12.

(46) Jiang, J.; Zhu, L.; Zhu, L.; Zhu, B.; Xu, Y. Surface characteristics of a self-polymerized dopamine coating deposited on hydrophobic polymer films. Langmuir 2011, 27 (23), 14180-14187.

(47) Huang, L.; Zhao, S.; Wang, Z.; Wu, J.; Wang, J.; Wang, S. In situ immobilization of silver nanoparticles for improving permeability, antifouling and anti-bacterial properties of ultrafiltration membrane. J. Membr. Sci. 2016, 499, 269-281.

(48) Yin, J.; Deng, B. Polymer-matrix nanocomposite membranes for water treatment. J. Membr. Sci. 2015, 479, 256-275.

(49) Tiraferri, A.; Yip, N. Y.; Straub, A. P.; Castrillon, S. R.-V.; Elimelech, M. A method for the simultaneous determination of transport and structural parameters of forward osmosis membranes. $J$. Membr. Sci. 2013, 444 (1), 523-538.

(50) Su, L.; Yu, Y.; Zhao, Y.; Liang, F.; Zhang, X. Strong antibacterial polydopamine coatings prepared by a shaking-assisted method. Sci. Rep. 2016, 6, 24420.

(51) Yu, B.; Liu, J.; Liu, S.; Zhou, F. Pdop layer exhibiting zwitterionicity: a simple electrochemical interface for governing ion permeability. Chem. Commun. 2010, 46 (32), 5900-5902.

(52) Van Houdt, R.; Michiels, C. W. Role of bacterial cell surface structures in Escherichia coli biofilm formation. Res. Microbiol. 2005, 156 (5-6), 626-633.

(53) Miller, D. J.; Araújo, P. A.; Correia, P. B.; Ramsey, M. M.; Kruithof, J. C.; van Loosdrecht, M. C. M.; Freeman, B. D.; Paul, D. R.; 
Whiteley, M.; Vrouwenvelder, J. S. Short-term adhesion and longterm biofouling testing of polydopamine and poly(ethylene glycol) surface modifications of membranes and feed spacers for biofouling control. Water Res. 2012, 46 (12), 3737-3753.

(54) Marambio-Jones, C.; Hoek, E. M. V. A review of the antibacterial effects of silver nanomaterials and potential implications for human health and the environment. J. Nanopart. Res. 2010, 12 (5), $1531-1551$ 